\title{
NEW GENERALISATIONS OF AN H-KKM TYPE THEOREM AND THEIR APPLICATIONS
}

\author{
Xie Ping Ding
}

\begin{abstract}
In this note, we establish some new generalisations of an H-KKM type theorem which unify and generalise the corresponding results of Horvath, BardaroCeppitelli, Tarafdar, Shioji, Park and others. As applications of our H-KKM type principle, we obtain some new generalisations of the Ky Fan type geometric properties of $H$-spaces, minimax inequalities and coincidence theorems in Horvath's abstract setting.
\end{abstract}

\section{INTRODUCTION}

The famous Fan-Knaster-Kuratowski-Mazurkiewicz theorem [14] has been generalised in various directions and has become an important and fundamental tool in treating many sophisticated nonlinear problems. Recently, Horvath [20, 21], BarbaroCeppitelli $[2,3]$, Ding $[11,12]$, Ding-Tan [13] and Tarafdar [43] generalised the FKKM theorem to $H$-spaces and gave applications in various fields.

Recently, Shioji [36] and Park [34] established some new KKM theorems involving an upper semicontinuous set-valued mapping with compact acyclic values.

In this note, we establish a new generalisation of the H-KKM theorem in Horvath's abstract setting which unifies and generalises the corresponding results mentioned above. As applications, we obtain some new generalisations of the Ky Fan type geometric properties of $H$-spaces, minimax inequalities and coincidence theorems in Horvath's abstract setting.

\section{Preliminaries}

Let $X$ and $Y$ be nonempty sets. We shall denote by $\mathcal{F}(X)$ the family of all nonempty finite subsets of $X$ and by $2^{Y}$ the family of all subsets of $Y$. Let $F: X \rightarrow 2^{Y}$ be a set-valued mapping. For $A \subset X$ and $y \in Y$, let

$$
F(A)=\bigcup\{F(x): x \in A\} \quad \text { and } \quad F^{-1}(y)=\{x \in X: y \in F(x)\} .
$$

Received 17 December 1992

The project supported by the National Natural Science Foundation of Peoples Republic of China,

Copyright Clearance Centre, Inc. Serial-fee code: 0004-9729/93 \$A2.00+0.00. 
For $B \subset Y$, the upper inverse of $B$ under $F$ is defined by

$$
F^{+}(B)=\{x \in X: \emptyset \neq F(x) \subset B\} \text {. }
$$

For topological spaces $X$ and $Y$, a subset $B$ of $Y$ is said to be compactly closed (respectively open) in $Y$ if for each compact subset $K$ of $Y$, the set $B \cap K$ is closed (respectively open) in $K$. An extended real-valued function $f: X \rightarrow \overline{\mathbb{R}}$ is lower semicontinuous (in short, l.s.c.) if the set $\{x \in X: f(x)>r\}$ is open in $X$ for each $r \in \overline{\mathbb{R}} ; f$ is called upper semi-continuous (in short, u.s.c.) if $-f$ is 1.s.c.. A mapping $F: X \rightarrow 2^{Y}$ is said to be u.s.c. if the set $F^{+}(V)$ is open in $X$ for each open subset $V$ of $Y$.

The following notions were introduced by Bardaro-Ceppitelli [2].

A pair $\left(X,\left\{\Gamma_{A}\right\}\right)$ is called an $H$-space if $X$ is a topological space and $\left\{\Gamma_{A}\right\}$ is a family of contractible subsets of $X$ indexed by $A \in \mathcal{F}(X)$ such that $\Gamma_{A} \subset \Gamma_{A^{\prime}}$, whenever $A \subset A^{\prime}$. A subset $D$ of an $H$-space $\left(X,\left\{\Gamma_{A}\right\}\right)$ is said to be

(i) $H$-convex if $\Gamma_{A} \subset D$ for each $A \in \mathcal{F}(D)$;

(ii) weakly $H$-convex if $\Gamma_{A} \cap D$ is contractible for each $A \in \mathcal{F}(D)$;

(iii) $H$-compact in $X$ if for each $A \in \mathcal{F}(X)$, there exists a compact, weakly $H$-convex subset $D_{A}$ of $X$ such that $D \cup A \subset D_{A}$.

Following Tarafdar [43], for a nonempty subset $D$ of an $H$-space $\left(X,\left\{\Gamma_{A}\right\}\right)$, we define the $H$-convex hull of $D$, denoted by $\mathrm{H}$-co $(D)$, as

$$
\mathrm{H}-\operatorname{co}(D)=\bigcap\{B \subset X: B \text { is } H \text {-convex and } D \subset B\} \text {. }
$$

It is easy to see that $\mathrm{H}-\operatorname{co}(D)$ is the smallest $H$-convex subset containing $D$ and we have

$$
\mathrm{H}-\operatorname{co}(D)=\bigcup\{\mathrm{H}-\operatorname{co}(A): A \in \mathcal{F}(D)\}
$$

by Lemma 1 of Tarafdar [43]. A mapping $F: D \rightarrow 2^{X}$ is said to be H-KKM if for each $A \in \mathcal{F}(D), \mathrm{H}-\operatorname{co}(A) \subset F(A)$.

Recall that a nonempty topological space is acyclic if all of its reduced Cech homology groups over the rationals vanish. In particular, any contractible space is acyclic, and hence any convex or star-shaped set in a topological vector space is acyclic. For a topological space $Y$, we shall denote by $k a(Y)$ the family of all compact acyclic subsets of $Y$.

Let $\left(X,\left\{\Gamma_{A}\right\}\right)$ be an $H$-space. For each $N \in \mathcal{F}(X), H-\operatorname{co}(N)$ is said to be a polytope in $X .\left(X,\left\{\Gamma_{A}\right\}\right)$ is said to be an $H$-space with compact polytopes if each polytope in $X$ is compact. If $X$ is a convex subset of a vector space with finite topology, then $X$ becomes a convex space (see, Lassonde [28]). For each $A \in \mathcal{F}(X)$, let $\Gamma_{A}=\operatorname{co}(A)$, then it is easy to see that $\left(X,\left\{\Gamma_{A}\right\}\right)$ becomes an $H$-space with compact polytopes. 
Let $\Delta_{n}$ be the standard $n$-dimensional simplex with vertices $e_{0}, \ldots, e_{n}$. If $J$ is a nonempty subset of $\{0, \ldots, n\}, \Delta_{J}$ will denote the convex hull of the vertices $\left\{e_{j}: j \in J\right\}$.

The following result is Lemma 1 of Ding-Tan in [13].

Lemma 2.1. Let $X$ be a topological space. For each nonempty subset $J$ of $\{0, \ldots, n\}$, let $\Gamma_{J}$ be a contractible subset of $X$. If $J \subset J^{\prime}$ implies $\Gamma_{J} \subset \Gamma_{J^{\prime}}$, then there exists a continuous mapping $f: \Delta_{n} \rightarrow X$ such that $f\left(\Delta_{J}\right) \subset \Gamma_{J}$ for each nonempty subset $J$ of $\{0, \ldots, n\}$.

The following result is Lemma 1 of Shioji in [36].

LEMMA 2.2. Let $\Delta_{n}$ be an $n$-dimensional simplex with the Euclidean topology and $W$ be a compact topological space. Let $\psi: W \rightarrow \Delta_{n}$ be a single-valued continuous mapping and $T: \Delta_{n} \rightarrow k a(W)$ be u.s.c.. Then there exists a point $x^{*} \in \Delta_{n}$ such that $x^{*} \in \psi\left(T\left(x^{*}\right)\right)$.

\section{Main Results}

In this section, we shall show some new generalisations of the H-KKM theorem.

Theorem 3.1. Let $D$ be a nonempty subset of an $H$-space $\left(X,\left\{\Gamma_{A}\right\}\right)$ with compact polytopes, $Y$ be a Hausdorff topological space, $G: D \rightarrow 2^{Y}$ and $T: H$-co $(D)$ $\rightarrow 2^{Y}$ such that

(1) for each $A \in \mathcal{F}(D),\left.T\right|_{z}: Z \rightarrow k a(Y)$ is u.s.c., where $Z=H$-co $(A)$,

(2) for each $A \in \mathcal{F}(D), T(H-c o(A)) \subset G(A)$,

(3) for each $A \in \mathcal{F}(D), G(x) \cap T(Z)$ is relatively closed in $T(Z)$ for each $x \in A$, where $Z=H-\operatorname{co}(A)$.

Then for any $A \in \mathcal{F}(D)$,

$$
T(H-\operatorname{co}(A)) \cap \bigcap_{x \in A} G(x) \neq \emptyset
$$

Proof: Suppose that the conclusion does not hold. Then there exists $A=$ $\left\{x_{0}, x_{1}, \ldots, x_{n}\right\} \in \mathcal{F}(D)$ such that

$$
T(Z) \cap \bigcap_{i=0}^{n} G\left(x_{i}\right)=\emptyset
$$

where $Z=\mathrm{H}-\operatorname{co}(A)$ is a compact polytope in $X$. Hence, we have

$$
T(Z) \subset T(Z) \backslash \bigcap_{i=1}^{n} G\left(x_{i}\right)=\bigcup_{i=1}^{n}\left(T(z) \backslash G\left(x_{i}\right)\right)
$$


For each $x \in D$, let $F(x)=T(Z) \backslash G(x)$, then we have

$$
T(Z) \subset \bigcup_{i=0}^{n} F\left(x_{i}\right)
$$

and each $F(x)$ is relatively open in $T(Z)$.

Since $Z$ is compact and $\left.T\right|_{z}: Z \rightarrow k a(Y)$ is u.s.c., it follows from Proposition 3.1.11 of Aubin-Ekeland [1] that $T(Z)$ is compact in $Y$. By (3.1), there exists a continuous parition of unity $\left\{\lambda_{i}\right\}_{i=0}^{n}$ subordinate to the open covering $\left\{F\left(x_{i}\right)\right\}_{i=0}^{n}$. Define a mapping $\psi: T(Z) \rightarrow \Delta_{n}$ by

$$
\psi(y)=\sum_{i=0}^{n} \lambda_{i}(y) e_{i} \quad \text { for each } \quad y \in T(Z) .
$$

Clearly, $\psi$ is continuous.

On the other hand, for each nonempty subset $J$ of $\{0, \ldots, n\}$, let $\Gamma_{J}=\Gamma_{\left\{x_{j}\right\}_{j \in J}}$, then $\Gamma_{J} \subset \mathrm{H}-\operatorname{co}(A)=Z$ is contractible and $J \subset J^{\prime}$ implies $\Gamma_{J} \subset \Gamma_{J^{\prime}}$. It follows from Lemma 2.1 that there exists a continuous mapping $f: \Delta_{n} \rightarrow Z$ such that

$$
f\left(\Delta_{J}\right) \subset \Gamma_{J}=\Gamma_{\left\{x_{j}\right\}_{j \in J}}
$$

By Theorem 7.3.11 of Klein-Thompson [25], the composition mapping $T \circ f: \Delta_{n} \rightarrow$ $k a(T(Z))$ is u.s.c.. It follows form Lemma 2.2 that there exists a point $x^{*} \in \Delta_{n}$ such that $x^{*} \in \psi\left(T\left(f\left(x^{*}\right)\right)\right)$. Let $y_{0} \in T\left(f\left(x^{*}\right)\right)$ be such that $x^{*}=\psi\left(y_{0}\right)$, then, by (3.2), we have

$$
x^{*}=\psi\left(y_{0}\right)=\sum_{i=0}^{n} \lambda_{i}\left(y_{0}\right) e_{i} .
$$

Let $J\left(y_{0}\right)=\left\{i \in\{0, \ldots, n\}: \lambda_{i}\left(y_{0}\right) \neq 0\right\}$, then

$$
x^{*}=\sum_{i \in J\left(y_{0}\right)} \lambda_{i}\left(y_{0}\right) e_{i} \in \Delta_{J\left(y_{0}\right)}
$$

and for each $i \in J\left(y_{0}\right), y_{0} \in F\left(x_{i}\right)$. Hence, we have

$$
y_{0} \notin \bigcup_{i \in J\left(y_{0}\right)} G\left(x_{i}\right)
$$

By (3.3) and (3.4), we have

$$
f\left(x^{*}\right) \in f\left(\Delta_{J\left(y_{0}\right)}\right) \subset \Gamma_{J\left(y_{0}\right)}=\Gamma_{\left\{x_{i}\right\}_{i \in J\left(y_{0}\right)}} \subset \mathrm{H}-\operatorname{co}\left(\left\{x_{i}\right\}_{i \in J\left(y_{0}\right)}\right) .
$$

It follows that

$$
y_{0} \in T\left(\mathrm{H}-\operatorname{co}\left(\left\{x_{i}\right\}_{i \in J\left(y_{0}\right)}\right)\right) \text {. }
$$

Properties (3.5) and (3.6) contradict the assumption (2). This completes our proof. 0 
REMARK 3.1. If $X=Y$ and $T$ is the identity mapping, condition (2) implies $G$ is an H-KKM mapping. If each $G(x)$ is compactly closed, condition (3) holds trivially. Theorem 3.1 generalises Theorem 1 of Shioji [36] to $H$-spaces.

THEOREM 3.2. Let $D$ be a nonempty subset of an $H$-space $\left(X,\left\{\Gamma_{A}\right\}\right)$ with compact polytopes, $Y$ be a Hausdorff topological space, $G: D \rightarrow 2^{Y}$ and $T: H-c o(D)$ $\rightarrow 2^{Y}$ such that

(1) for each $x \in D, G(x)$ is compactly closed,

(2) for each $A \in \mathcal{F}(D), T(H$-co $(A)) \subset G(A)$,

(3) for each $A \in \mathcal{F}(D),\left.T\right|_{Z}: Z \rightarrow k a(Y)$ is u.s.c., where $Z=H$-co $(A)$,

(4) there exists a nonempty compact subset $K$ of $Y$ such that for each $A \in$ $\mathcal{F}(D), T(H-\operatorname{co}(A)) \subset K$.

Then $K \cap \bigcap_{x \in D} G(x) \neq \emptyset$.

Proof: By Theorem 3.1 and condition (4), the family $\{G(x) \cap K: x \in D\}$ has the finite intersection property. Since $K$ is compact, the conclusion holds.

REMARK 3.2. Theorem 3.2 improves and generalises Theorem 2 of Shioji [36] to $H$ spaces. By the way, we point out that the condition $T(X) \subset K$ in Theorem 2 of Shioji [36] should be replaced by $T(\operatorname{co}(X)) \subset K$, otherwise the conclusion does hot hold.

THEOREM 3.3. Let $D$ be nonempty subset of an $H$-space $\left(X,\left\{\Gamma_{A}\right\}\right)$ with compact polytopes, $Y$ be a Hausdorff topological space, $G: D \rightarrow 2^{Y}$ and $T: H-c o(D) \rightarrow$ $k a(Y)$ be u.s.c. such that

(1) for each $x \in D, G(x)$ is compactly closed,

(2) for each $A \in \mathcal{F}(D), T(H-c o(A)) \subset G(A)$.

Furthermore suppose that one of the following conditions is satisfied:

(3) $H-c o(D)$ is compact, or

(4) there exist an $H$-compact subset $L$ of $X$ and a nonempty compact subset $K$ of $Y$ such that for each $N \in \mathcal{F}(D)$ and for each $y \in T\left(L_{N}\right) \backslash K$, there is an $x \in L_{N} \cap D$ such that $y \notin T\left(L_{N}\right) \cap G(x)$.

Then $\operatorname{cl}(T(H-c o(D))) \cap K \cap \bigcap_{x \in D} G(x) \neq \emptyset$.

Proof: First suppose that condition (3) is satisfied. Since $H-\operatorname{co}(D)$ is compact and $T: \mathrm{H}-\operatorname{co}(D) \rightarrow k a(Y)$ is u.s.c., it follows from Proposition 3.1.11 of Aubin-Ekeland [1] that $T(\mathrm{H}-\operatorname{co}(D))$ is compact in $Y$. Let $K=T(\mathrm{H}-\mathrm{co}(D))$, then the conclusion holds from Theorem 3.2 .

Next suppose that condition (4) is satisfied. It is easy to see that condition (4) is equivalent to the following condition:

(4)' there exist an $\boldsymbol{H}$-compact subset $L$ of $X$ and a nonempty compact subset 
$K$ of $Y$ such that for each $N \in \mathcal{F}(D)$,

$$
T\left(L_{N}\right) \cap \bigcap_{x \in L_{N} \cap D} G(x) \subset K .
$$

Note that for each $N \in \mathcal{F}(D),\left(L_{N},\left\{\Gamma_{A} \cap L_{N}\right\}\right)$ is a compact $H$-space and $L \cup N \subset$ $L_{N}$. By applying Theorem 3.2 with $(D, X, Y, K, G, T)$ instead of $\left(L_{N} \cap D, L_{N}\right.$, $\left.Y, T\left(L_{N}\right),\left.G\right|_{L_{N} \cap D},\left.T\right|_{L_{N}}\right)$, we have

$$
T\left(L_{N}\right) \cap \bigcap_{x \in L_{N} \cap D} G(x) \neq \emptyset
$$

and hence, by condition (4)',

$$
T\left(L_{N}\right) \cap K \cap \bigcap_{x \in L_{N} \cap D} G(x) \neq 0 .
$$

Since $N \subset L_{N}$, it follows that

$$
\operatorname{cl}(T(\mathrm{H}-\mathrm{co}(D))) \cap K \cap \bigcap_{x \in N} G(x) \neq \emptyset .
$$

This shows that the family $\{\operatorname{cl}(T(\mathrm{H}-\operatorname{co}(D))) \cap K \cap G(x): x \in D\}$ has the finite intersection property. Since $\mathrm{cl}(T(\mathrm{H}-\mathrm{co}(D))) \cap K$ is compact in $Y$, the conclusion holds.

REMARK 3.3. We note that condition (a) of Theorem 3 of Shioji [36] should be replaced by the condition that $X$ is compact and convex, otherwise his Theorem 2 cannot be applied. Theorem 3.3 improves and generalises Theorem 3 of Shioji [36] to $H$-spaces. For $X=Y$ and $T=I$, the identity mapping, condition (2) implies that $G$ is H-KKM and hence Theorem 3.3 generalises Theorem 1 of Bardaro-Ceppitelli [2] and Corollary 1 of Horvath [20]. If $T=s: X \rightarrow Y$ is a single-valued continuous mapping and $X$ is a convex space, Theorem 3.3 also generalises Park [32, Theorems 3 and 4], [33, Theorem 4]; Chang [9, Theorem 2.1]; Lassonde [28, Theorems I and III] and Fan [14, Lemma 1], [16, Theorem 1], [17, Theorem 4].

The following result is an consequence of Theorem 3.3.

TheOREM 3.4. Let $D$ be a nonempty subset of an $H$-space $\left(X,\left\{\Gamma_{A}\right\}\right)$ with compact polytopes, $Y$ be a Hausdorff topological space, $G: D \rightarrow 2^{Y}$ and $T: H-c o(D)$ $\rightarrow k a(Y)$ be u.s.c. such that

(1) for each $x \in D, G(x)$ is compactly closed,

(2) for each $A \in \mathcal{F}(D), T(H-\operatorname{co}(A)) \subset G(A)$,

(3) there exist an $H$-compact subset $L$ of $X$ and a nonempty compact subset $K$ of $Y$ such that for each $N \in \mathcal{F}(D), x \in L_{N} \backslash T^{+}(K)$ implies

$$
\bigcap\left\{G(z): z \in L_{N} \cap D\right\} \subset Y \backslash T(x) .
$$


Then $\operatorname{cl}(T(H-\operatorname{co}(D))) \cap K \cap\{G(x): x \in D\} \neq \emptyset$.

Proof: To prove the conclusion, it suffices to show that condition (3) implies condition (4) of Theorem 3.3. In fact, if condition (4) of Theorem 3.3 does not hold, then for any $H$-compact subset $L$ of $X$ and for any nonempty compact subset $K$ of $Y$, there exist $N \in \mathcal{F}(D)$ and $y \in T\left(L_{N}\right) \backslash K$ such that for all $x \in L_{N} \cap D$, $y \in T\left(L_{N}\right) \cap G(x)$ and hence $y \in T\left(L_{N}\right) \cap\left\{G(z): z \in L_{N} \cap D\right\}$. Since $y \in T\left(L_{N}\right) \backslash K$, there exists $x \in L_{N}$ such that $y \in T(x) \backslash K$, therefore $x \in L_{N} \backslash T^{+}(K)$ and

$$
\bigcap\left\{G(z): z \in L_{N} \cap D\right\} \not \subset Y \backslash T(x)
$$

since $y \in T(x) \cap\left\{G(z): z \in L_{N} \cap D\right\}$. This shows that condition (3) does not hold and completes our proof.

Remark 3.4. Theorem 3.4 generalises Theorem 3 of Park [34] and many known KKM type theorems in the literature. For example, see the particular forms of Theorem 3 of Park [34].

\section{Some applications}

In this section, we shall give some applications of our H-KKM type theorems to the geometric properties of $\boldsymbol{H}$-spaces, coincidence theorems and minimax inequalities in $\boldsymbol{H}$-spaces.

THEOREM 4.1. Let $D$ be a nonempty subset of an $H$-space $\left(X,\left\{\Gamma_{A}\right\}\right)$ with compact polytopes, $Y$ be a Hausdorff topological space and $A \subset B \subset C \subset H-c o(D) \times Y$ such that $A$ is nonempty closed in $H-c o(D) \times Y$. Suppose that

(1) for each $x \in D,\{y \in Y:(x, y) \in C\}$ is compactly closed,

(2) for each $y \in Y,\{x \in D:(x, y) \notin B\}$ is empty or $H$-convex,

(3) there exist a nonempty compact subset $K$ of $Y$ such that for $x \in$ $H$-co $(D)$, the set $\{y \in K:(x, y) \in A\}$ is acyclic.

Then there exists a point $y_{0} \in K$ such that $D \times\left\{y_{0}\right\} \subset C$.

Proof: Define the mappings $H, G: D \rightarrow 2^{Y}$ and $T: H-\operatorname{co}(D) \rightarrow 2^{Y}$ by

and

$$
\begin{aligned}
& H(x)=\{y \in Y:(x, y) \in B\} \\
& G(x)=\{y \in Y:(x, y) \in C\} \\
& T(x)=\{y \in K:(x, y) \in A\} .
\end{aligned}
$$

For each $x \in D, G(x)$ is compactly closed by (1). Since $A$ is closed in $\mathrm{H}-\operatorname{co}(D) \times Y$, each $T(x)$ is closed and the graph of $T$ is closed. From Corollary 3.1.9 of Aubin-Ekeland [1] it follows that $T$ is u.s.c. and hence $T: \mathrm{H}-\operatorname{co}(D) \rightarrow k a(Y)$ is u.s.c. by (3). We 
claim that for each $N \in \mathcal{F}(D), T(\mathrm{H}-\operatorname{co}(N)) \subset H(N)$. If it is not true, then there exist $N \in \mathcal{F}(D)$ and $y \in T(\mathrm{H}-\operatorname{co}(N))$ such that $y \notin H(N)$ and hence we have

$$
N \subset\{x \in D:(x, y) \notin B\} .
$$

By (2), we have $\mathrm{H}-\operatorname{co}(N) \subset\{x \in D:(x, y) \notin B\} \subset\{x \in \mathrm{H}-\operatorname{co}(D):(x, y) \notin A\}=$ H-co $(D) \backslash\{x \in H-c o(D):(x, y) \in A\}=\mathrm{H}-\operatorname{co}(D) \backslash T^{-1}(y)$. It follows that $y \notin$ $T(\mathrm{H}-\operatorname{co}(N))$, which is a contradiction. Hence for each $N \in \mathcal{F}(D), T(\mathrm{H}-\operatorname{co}(N)) \subset$ $H(N) \subset G(N)$. By (3), there exists a nonempty compact subset $K$ of $Y$ such that $T(\mathrm{H}-\operatorname{co}(D)) \subset K$. By applying Theorem 3.2, we have $K \cap \cap\{G(x): x \in D\} \neq \emptyset$. This implies that there exists a point $y_{0} \in K$ such that $D \times\left\{y_{0}\right\} \subset C$.

Remark 4.1. Theorem 4.1 improves and generalises Park [34, Theorem 10 and Corollary 10.1], Shioji [36, Corollary 1]; Ha [18, Theorem 3] and Fan [14, Lemma 4] to $H$-spaces.

THEOREM 4.2. Let $D$ be a nonempty subset of an $H$-space $\left(X,\left\{\Gamma_{A}\right\}\right)$ with compact polytopes, $Y$ be a Hausdorff topological space, $F, S: D \rightarrow 2^{Y}$ and $T: H$-co(D) $\rightarrow k a(Y)$ be u.s.c. such that

(1) for each $A \in \mathcal{F}(D), F(x) \cap T(Z)$ is relatively open in $T(Z)$ for each $x \in A$ where $Z=H$-co $(A)$,

(2) for each $A \in \mathcal{F}(D)$ and for each $y \in T(H-c o(A)), A \in \mathcal{F}\left(F^{-1}(y)\right)$ implies $H-\operatorname{co}(A) \subset S^{-1}(y)$,

(3) there exists an $N \in \mathcal{F}(D)$ such that $T(H-c o(N)) \subset F(N)$.

Then there exists a point $x_{0} \in D$ such that $T\left(x_{0}\right) \cap S\left(x_{0}\right) \neq \emptyset$.

Proof: Define a mapping $G: D \rightarrow 2^{Y}$ by $G(x)=Y \backslash F(x)$ for each $x \in D$. Then for each $x \in D$ and for each $A \in \mathcal{F}(D), G(x) \cap T(Z)$ is relatively closed in $T(Z)$ where $Z=\mathrm{H}-\operatorname{co}(A)$ by (1). By (3), there exists an $N \in \mathcal{F}(D)$ such that

$$
T(\mathrm{H}-\operatorname{co}(N)) \subset \bigcup_{x \in N} F(x)=\bigcup_{x \in N}(Y \backslash G(x))=Y \backslash \bigcup_{x \in N} G(x)
$$

and hence $T(\mathrm{H}-\operatorname{co}(N)) \cap \bigcap_{x \in N} G(x)=\emptyset$. Therefore the conclusion of Theorem 3.1 does not hold. It follows that condition (2) of Theorem 3.1 must not hold. Hence there exists an $A \in \mathcal{F}(D)$ such that $T(\mathrm{H}-\operatorname{co}(A)) \not \subset G(A)$, that is there exist $y \in T(\mathrm{H}-\operatorname{co}(A))$ and $x_{0} \in \mathrm{H}-\operatorname{co}(A)$ such that $y \in T\left(x_{0}\right)$ and

$$
y \notin G(A)=\bigcup_{x \in A}(Y \backslash F(x))=Y \backslash \bigcap_{x \in A} F(x) .
$$

Hence, we have $y \in \bigcap_{x \in A} F(x)$ and $A \in \mathcal{F}\left(F^{-1}(y)\right)$. By (2), we have

$$
x_{0} \in \mathrm{H}-\operatorname{co}(A) \subset S^{-1}(y)
$$


and hence $y \in T\left(x_{0}\right) \cap S\left(x_{0}\right)$. This completes the proof.

TheOREM 4.3. Let $D$ be a nonempty subset of an $H$-space $\left(X,\left\{\Gamma_{A}\right\}\right)$ with compact polytopes, $Y$ be a Hausdorf topological space, $F, S: D \rightarrow 2^{Y}, T: H-c o(D) \rightarrow$ $k a(Y)$ be u.s.c. and $K$ be a nonempty compact subset of $Y$ such that

(1) for each $x \in D, F(x)$ is compact open,

(2) for each $A \in \mathcal{F}(D)$ and for each $y \in T(H-c o(A)), A \in \mathcal{F}\left(F^{-1}(y)\right)$ implies $H-\operatorname{co}(A) \subset S^{-1}(y)$,

(3) $\operatorname{cl}(T(H-\operatorname{co}(D))) \cap K \subset F(D)$,

(4) there exists an $H$-compact subset $L$ of $X$ such that for each $N \in \mathcal{F}(D)$, $x \in L_{N} \backslash T^{+}(K)$ implies $T(x) \subset F\left(L_{N} \cap D\right)$.

Then there exists a poiot $x_{0} \in D$ such that $T\left(x_{0}\right) \cap S\left(x_{0}\right) \neq 0$.

Proof: Since $\mathrm{cl}(T(\mathrm{H}-\operatorname{co}(D))) \cap K$ is compact and covered by compactly open sets $\{F(x)\}_{x \in D}$ by (1) and (3), there exists $N_{1} \in \mathcal{F}(D)$ such that

$$
\operatorname{cl}(T(\mathrm{H}-\operatorname{co}(D))) \cap K \subset F\left(N_{1}\right) \text {. }
$$

Consider the set $L_{N_{1}}$ in (4). We claim that $T\left(L_{N_{1}}\right) \subset F\left(L_{N_{1}} \cap D\right)$. In fact, if $x \in$ $L_{N_{1}} \cap T^{+}(K)$, then $T(x) \subset K$ and

$$
T(x) \subset T\left(L_{N_{1}}\right) \cap K \subset T(\mathrm{H}-\operatorname{co}(D)) \cap K \subset F\left(N_{1}\right) \subset F\left(L_{N_{1}} \cap D\right) .
$$

On the other hand, if $x \in L_{N_{1}} \backslash T^{+}(K)$, then $T(x) \subset F\left(L_{N_{1}} \cap D\right)$ by (4). Hence, we have $T\left(L_{N_{1}}\right) \subset F\left(L_{N_{1}} \cap D\right)$.

Since $L_{N_{1}}$ is compact and $T: \mathrm{H}-\operatorname{co}(D) \rightarrow k a(Y)$ is u.s.c., therefore $T\left(L_{N_{1}}\right)$ is compact and included in $F\left(L_{N_{1}} \cap D\right)$. By (1), there exists $N \in \mathcal{F}\left(L_{N_{1}} \cap D\right)$ such that $T\left(L_{N_{1}}\right) \subset F(N)$. Note that $\left(L_{N_{1}},\left\{\Gamma_{A} \cap L_{N_{1}}\right\}\right)$ is an $H$-space and $N \in \mathcal{F}\left(L_{N_{1}}\right)$, so we have

$$
\mathrm{H}-\operatorname{co}(N) \subset L_{N_{1}} \text { and } T(\mathrm{H}-\operatorname{co}(N)) \subset T\left(L_{N_{1}}\right) \subset F(N) \text {. }
$$

Thus, condition (3) of Theorem 4.2 is satisfied. The conclusion holds from Theorem 4.2 .

REMARK 4.2. Theorem 4.3 improves and generalises Theorem 1 of Park [34] to $H$ spaces. As particular forms of Theorem 4.3, we easily obtain the following results: Ding [11, Theorem 2.1]; Chang-Ma [10, Theorem 7 and Corollary 4]; Park [34, Corollary 1.1], [32, Theorem 6], [33, Theorem 7]; Chang [9, Theorems 2.4 and 2.7]; Browder [6, Theorems 1 and 7], [7, Proposition 1], [8, Theorems 2 and 5]; Tarafdar [39, Theorem 1], [40, Corollary 2.1 and Theorem 2.2], [41, Theorem 1.2], [42, Theorem 2]; TarafdarHusain [44, Theorem 1.1]; Ben-El-Mechaiekh-Deguire-Granas [4, Théorème 1], [5, I, Théorème 1.2 et 5; II, Théorèmes 3.1-3.3 et 4.1]; Yanaelis-Prabhakar [45, Theorems 3.2 
and 3.3]; Lassonde [28, Theorem 1.1]; Ko-Tan [26, Theorem 3.1]; Simons [37, Theorem 4.3]; Takahashi [38, Theorems 2 and 5]; Komiya [27, Theorem 1]; Mehta [30, Theorem 3.1]; Mehta-Tarafdar [31, Theorem 1-5]; Sessa [35, Theorems 4, 7 and 8]; Jiang [22, I, Lemma 3.2], [23, Lemma 2.1], [24, Corollary 3.2] and McLinden [29, Theorem].

TheOREM 4.4. Let $D$ be a nonempty subset of an $H$-space $\left(X,\left\{\Gamma_{A}\right\}\right)$ with compact polytopes, $Y$ be a Hausdorff topological space and $T: H$-co $(D) \rightarrow k a(Y)$ be u.s.c. Let $M$ and $N$ be subsets of a set $Z, f, g: D \times Y \rightarrow Z$ and $K$ be a nonempty compact subset of $Y$. Suppose that

(1) for each $x \in D$, the set $\{y \in Y: g(x, y) \in M\}$ is compactly open,

(2) for each $A \in \mathcal{F}(D)$ and for each $y \in T(H-c o(A))$, $A \in \mathcal{F}(\{x \in D: g(x, y) \in M\})$ implies $H$-co $(A) \subset\{x \in D: f(x, y) \in N\}$,

(3) there exists an $H$-compact subset $L$ of $X$ such that for each $A \in \mathcal{F}(D)$, $x \in L_{A} \backslash T^{+}(K)$ and $y \in T(x)$, there exists an $x_{1} \in L_{A}$ satisfying $g\left(x_{1}, y\right) \in M$.

Then either

(a) there exists an $y^{*} \in \operatorname{cl}(T(H-c o(D))) \cap K$ such that $g\left(x, y^{*}\right) \notin M$ for all $x \in D$, or

(b) there exist $x^{*} \in D$ and $y^{*} \in T\left(x^{*}\right)$ such that $f\left(x^{*}, y^{*}\right) \in N$.

Proof: Define the mappings $F, S: D \rightarrow 2^{Y}$ by

$$
F(x)=\{y \in Y: g(x, y) \in M\} \text { and } S(x)=\{y \in Y: f(x, y) \in N\}
$$

for each $x \in D$. Then conditions (1) and (2) of Theorem 4.3 are satisfied by (1) and (2). Suppose that conclusion (a) does not hold, then $\operatorname{cl}(T(\mathrm{H}-\mathrm{co}(D))) \cap D \subset F(D)$ and condition (3) of Theorem 4.3 is satisfied. It is easy to see that condition (3) implies condition (4) of Theorem 4.3. By Theorem 4.3, there exists a point $x^{*} \in D$ such that $T\left(x^{*}\right) \cap S\left(x^{*}\right) \neq \emptyset$, that is, there exists $y^{*} \in T\left(x^{*}\right)$ such that $f\left(x^{*}, y^{*}\right) \in N$.

REMARK 4.3. Theorem 4.4 improves and generalises Theorem 2.4 of Ding [12], Theorem 5 of Park [34] and many known results in the literature, see the particular forms of Theorem 5 of Park [34]. From Theorem 4.4, we easily state its analytic alternative which generalises Theorem 6 of Park [34]. We omit the statement.

Let $\left(X,\left\{\Gamma_{A}\right\}\right)$ be an $H$-space. Recall that a real-valued function $f: X \rightarrow \mathbb{R}$ is said to be $H$-quasiconcave if for each $t \in R$, the set $\{x \in X: f(x)>t\}$ is $H$-convex.

TheOREM 4.5. Let $D$ be a nonempty subset of an $H$-space $\left(X,\left\{\Gamma_{A}\right\}\right)$ with compact polytopes, $Y$ be a Hausdorff topological space and $T: H-c o(D) \rightarrow k a(Y)$ be u.s.c.. Suppose that two functions $f, g: D \times Y \rightarrow \mathbb{R} \cup\{+\infty\}$ satisfy the following conditions:

(1) $g(x, y) \leqslant f(x, y)$ for all $(x, y) \in D \times Y$, 
(2) for each $x \in D, y \rightarrow g(x, y)$ is 1.s.c. on each compact subset of $Y$,

(3) for each $y \in T(H-\operatorname{co}(D)), x \rightarrow f(x, y)$ is $H$-quasiconcave on $H-c o(D)$,

(4) there exists an $H$-compact subset $L$ of $X$ such that for each $t \in \mathbb{R}$, each $N \in \mathcal{F}(D)$, each $x \in L_{N} \backslash T^{+}(K)$ and each $y \in T(x)$, there exists $x_{1} \in L_{N}$ satisfying $g\left(x_{1}, y\right)>t$.

Then

(a) there exists an $y^{*} \in \operatorname{cl}(T(H-\operatorname{co}(D))) \cap K$ such that

$$
\sup _{x \in D} g\left(x, y^{*}\right) \leqslant \sup _{(x, y) \in G \mathbf{r}(T)} f(x, y)
$$

and

(b) the following minimax inequality holds:

$$
\min _{y \in K} \sup _{x \in D} g(x, y) \leqslant \sup _{(x, y) \in G_{r}(T)} f(x, y)
$$

where $\operatorname{Gr}(T)=\{(x, y) \in H-\operatorname{co}(D) \times Y: y \in T(x)\}$ is the graph of $T$.

Proof: It is obvious that conclusion (a) implies conclusion (b). In order to show (a) we may assume that $t=\sup \{f(x, y):(x, y) \in \operatorname{Gr}(T)\}$ is finite. In Theorem 4.4, put $Z=\overline{\mathbb{R}}, M=N=(t,+\infty)$. Then, by (2), condition (1) of Theorem 4.4 is satisfied. It is easy to check that conditions (1) and (3) imply condition (2) of Theorem 4.4 and condition (4) implies condition (3) of Theorem 4.4. Obviously, conclusion (b) of Theorem 4.4 does not hold. Hence we conclude that the conclusion (a) of Theorem 4.4 holds. This completes our proof.

REMARK 4.4. Theorem 4.5 improves and generalises Theorem 9 of Park [34], Theorem 1 of $\mathrm{Ha}$ [19], Theorem 1 of Fan [15] and many known minimax inequalities in the literature to $H$-spaces, see the particular forms of Theorem 9 of Park [34].

\section{REFERENCES}

[1] J.P. Aubin and I. Ekeland, Applied nonlinear analysis (John Wiley and Sons, New York, 1984).

[2] C. Bardaro and R. Ceppitelli, 'Some further generalizations of Knaster-KuratowskiMazurkiewicz theorem and minimax inequalities', J. Math. Anal. Appl. 132 (1988), 484-490.

[3] C. Bardaro and R. Ceppitelli, 'Applications of the generalized Knaster-KuratowskiMazurkiewicz theorem to variational inequalities', J. Math. Anal. Appl. 137 (1989), 46-58.

[4] H. Ben-El-Mechaiekh, P. Deguire and A. Granas, 'Une alternative non linéaire en analyse convexe et applications', C.R. Acad. Sci. Paris 295 (1982), 257-259. 
[5] H. Ben-El-Mechaiekh, P. Deguire and A. Granas, 'Points fixes et coincidences pour les applications multivoque', C.R. Acad. Sci. Paris 295 (1982), I,337-340; II,381-384.

[6] F.E. Browder, "The fixed point theory of multi-valued mappings in topological vector spaces', Math. Ann. 177 (1968), 283-301.

[7] F.E. Browder, 'On a sharpened form of the Sthauder fixed-point theorem theorem', Proc. Nat. Acad. Sci. U.S.A. 74 (1977), 4749-4751.

[8] F.E. Browder, 'Coincidence theorems, minimax theorems, and variational inequalities', Contemp. Math. 26 (1984), 67-80.

[9] S.Y. Chang, 'A generalization of KKM principle and its applications', Soochow J. Math. 15 (1989), 7-17.

[10] S.S. Chang and Y.H. Ma, 'Generalized KKM theorem on $H$-space with applications', $J$. Math. Anal. Appl. 163 (1992), 406-421.

[11] X.P. Ding, 'Coincidence theorems in $H$-spaces and its applications', J. Sichuan Normal Univ. 14 (1991), 27-32.

[12] X.P. Ding, 'Existence theorem of maximizable $H$-quasiconcave funcations', Acta Math. Sinica 36 (1993), 273-279.

[13] X.P. Ding and K.K. Tan, 'Matching theorems, fixed point theorems and minimax inequalities without convexity', J. Austral. Math. Soc. Ser. A 49 (1990), 111-128.

[14] Ky Fan, 'A generalization of Tychonoff's fixed point theorem', Math. Ann. 142 (1961), 305-310.

[15] Ky Fan, 'A minimax inequality and applications', in Inequalities III, (O. Shisha, Editor) (Academic Press, New York, 1972), pp. 103-113.

[16] Ky Fan, 'Fixed-point and related theorems for noncompact convex sets', in Game theory and related topics, (O. Moeshlin and D. Pallaschke, Editors) (North-Holland, Amsterdam, 1979), pp. 151-156.

[17] Ky Fan, 'Some properties of convex sets related fixed point theorems', Math. Ann. 266 (1984), 519-537.

[18] C.W. Ha, 'Minimax and fixed point theorems', Math. Ann. 248 (1980), 73-77.

[19] C.W. Ha, 'On a minimax inequality of Ky Fan', Proc. Amer. Math. Soc. 99 (1987), 680-682.

[20] C. Horvath, 'Some results on multivalued mappings and inequalities without convexity', in Nonlinear and convex analysis, (B.L. Lin and S. Simons, Editors) (Dekker, New York, 1987), pp. 99-106.

[21] C. Horvath, 'Contractibility and generalized convexity', J. Math. Anal. Appl. 156 (1991), 341-357.

[22] J. Jiang, 'Fixed point theorems for paracompact convex sets', Acta Math. Sinica 4 (1988), I, 64-71; II, 234-241.

[23] J. Jiang, 'Fixed point theorems for convex set', Acta Math. Sinica 4 (1988), 356-363.

[24] J. Jiang, 'Coincidence theorems and minimax theorems', Acta Math. Sinica 5 (1989), 307-320.

[25] E. Klein and A.C. Thompson, Theory of correspondence (John Wiley and Sons, New York, 
1984).

[26] H.M. Ko and K.K. Tan, 'A coincidence theorem with applications to minimax inequalities and fixed point theorems', Tamkang J. Math. 17 (1986), 37-45.

[27] H. Komiya, 'Coincidence theorems and saddle point theorem', Proc. Amer. Math. Soc. $\theta 6$ (1986), 599-602.

[28] M. Lassonde, 'On the use of KKM multifunctions in fixed point theory and related topics', J. Math. Anal. Appl. 97 (1983), 151-201.

[29] L. McLinden, 'Acyclic multifunctions without metrizability', in Résumés des Colloque International: Théorie du Point Fixe et Applications (Marseille-Luminy, 1989), pp. 150-151.

[30] G. Mehta, 'Fixed points equilibria and maximal elements in linear topological spaces', Comment. Math. Univ. Carolin. 28 (1987), 377-385.

[31] G. Mehta and E. Tarafdar, 'Infinite-dimensional Gale-Nikaido-Debreu theorem and a fixed point theorem of Tarafdar', J. Econom. Theory. 41 (1987), 333-339.

[32] S. Park, 'Generalizations of Ky Fan's matching theorems and their applications', J. Math. Anal. Appl. 141 (1989), 164-176.

[33] S. Park, 'Generalizations of Ky Fan's matching theorems and their applications II', $J$. Korean Math. Soc. 28 (1991), 275-283.

[34] S. Park, 'Some coincidence theorems on acyclic multifunctions and applications to KKM theory', in Fixed point theory and applications, (K.K. Tan, Editor) (World Sci. Pub. Co. Pte. Ltd., Singapore, 1992), pp. 248-277.

[35] S.Sessa, 'Some remarks and applications of an extension of a lemma of Ky Fan', Comment. Math. Univ. Carolin. 29 (1988), 567-575.

[36] N. Shioji, 'A further generalization of the Knaster-Kuratowski-Mazurkiewicz theorem', Proc. Amer. Math. Soc. 111 (1991), 187-195.

[37] S. Simons, 'Two-function minimax theorems and variational inequalities for functions on compact and noncompact sets, with some comments on fixed-point theorems', Proc. Sympos. Pure Math. 45 (1986), 377-392.

[38] W. Takahashi, 'Fixed point, minimax, and Hahn-Banach theorems', Proc. Sympos. Pure Math. 45 (1986), 419-427.

[39] E. Tarafdar, 'On nonlinear variational inequalities', Proc. Amer. Math. Soc. 67 (1977), 95-98.

[40] E. Tarafdar, 'On minimax principle and sets with convex sections', Publ. Math. Debrecen 29 (1982), 219-226.

[41] E. Tarafdar, 'Variational problems via a fixed point theorem', Indian J. Math. 28 (1986), 229-240.

[42] E. Tarafdar, 'A fixed point theorem equivalent to the Fan-Knaster-KuratowskiMazurkiewicz theorem', J. Math. Anal. Appl. 63 (1987), 475-470.

[43] E. Tarafdar, 'A fixed point theorem in $H$-space and related results', Bull. Austral. Math. Soc. 42.(1990), 133-140.

[44] E. Tarafdar and T. Husain, 'Duality in fixed point theory of multi-valued mappings with applications', J. Math. Anal. Appl. 63 (1978), 371-376. 
[45] N. Yannelis and N.D. Prabhakar, 'Existence of maximal elements and equilibria in linear topological spaces', J. Math. Econom. 12 (1983), 233-245.

Department of Mathematics

Sichuan Normal University

Chengdu

Sichuan 610066

Peoples Republic of China 